\title{
Use of an immunodominant p17 antigenic fraction of Neospora caninum in detection of antibody response in cattle
}

\author{
Gema Álvarez García, Inmaculada López Pérez, Elisabeth Innes*, \\ Esther Collantes Fernandez, Aurora Fernandez Garcia, Mercedes Gomez Bautista, \\ Luis Miguel Ortega Mora/ ${ }^{+}$
}

\begin{abstract}
Departamento de Sanidad Animal, Facultad de Veterinaria, Universidad Complutense de Madrid, Avd. Puerta de Hierro s/n, 28040-Madrid, España *Moredun Research Institute, Pentlands Science Park, Edinburgh, UK
\end{abstract}

\begin{abstract}
A Neospora caninum $17 \mathrm{kDa}$ protein fraction (p17) has been described as an immunodominant antigen (IDA) under reducing and non-reducing conditions. The aim of the present study was to investigate the diagnostic utility of p17 in cattle. In order to achieve this, p17 was purified by electroelution from whole $\mathrm{N}$. caninum tachyzoite soluble extract and a p17-based Western blot (WB-p17) was developed. The p17 recognition was measured by densitometry and expressed as $O D$ values to check the validity of the WB-p17. A total of 131 sera including sequential samples from naturally-and experimentally-infected calves and breeding cattle were analysed by WB-p17 and compared with IFAT using whole formalin-fixed tachyzoites as a reference test. The results obtained highlight the feasibility of using the N. caninum p17 in a diagnostic test in cattle. Firstly, the assay based on the p-17 antigen discriminated between known positive and negative sera from different cattle populations, breeding cattle and calves. Secondly, the p17 antigen detected fluctuations in the antibody levels and seroconversion in naturally-and experimentally-infected cattle. Significant differences in p-17 antigen recognition were observed between naturally infected aborting and non-aborting cattle, as well as significant antibody fluctuations over time in experimentally infected cattle, which varied between groups. Furthermore, the results obtained with WB-p17 are in accordance with the results obtained with the IFAT, as high agreement values were obtained when all bovine subpopulations were included $(\kappa=0.86)$.
\end{abstract}

Key words: antibody response - Neospora caninum - cattle - Western bolt-p17

Neospora caninum, a cyst-forming coccidian parasite, has been identified as an important cause of abortion and neonatal mortality in cattle worldwide (Dubey 1999). Infection of animals with $N$. caninum elicits the development of characteristic immunoglobulin $\mathrm{G}(\mathrm{IgG})$ antibody responses against low molecular weight tachyzoite antigens (Bjerkas et al. 1994).

Preliminary work has suggested the $17 \mathrm{kDa}$ immunodominant antigent as a possible antigenic marker since changes in its recognition could be related with acute infection or reactivation of a chronic infection (AlvarezGarcia et al. 2002). The recognition of the $\mathrm{p} 17$ antigenic fraction is very specific for N. caninum infection and rabbit hyperimmune serum against Toxoplasma gondii did not react with this protein (Louie et al. 1997).

Therefore, the $\mathrm{p} 17$ antigenic fraction (p17) was selected to evaluate its potential as a diagnostic tool. To achieve this, a Western blot (WB) based on this antigen (WBp17) was developed for the detection and quantification

Financial support: the European Commission (QRLT-200001050). This work was also part of the EU research collaboration COST-854. GA was financed by a grant from Consejería de Educación de la Comunidad de Madrid, España

Corresponding author: luis.ortega@vet.ucm.es

Received 2 January 2006

Accepted 31 May 2006 of serum $\operatorname{IgG}$ antibodies from naturally-and experimentally-infected cattle. All samples were also analysed by IFAT, which was regarded as the reference technique.

\section{MATERIALS AND METHODS}

Sera - Sera were collected from cattle naturally- and experimentally-infected with $N$. caninum. Serum samples from naturally-infected bovine animals were analysed and divided into three groups based on clinical findings and serological profiles - infected aborting and non-aborting breeding cattle as well as non-infected breeding cattle as previously described (Alvarez-Garcia et al. 2003) (Table I). Cattle came from herds with a previous history of Neospora abortion problems in the last three years and proved to be seropositive by IFAT test.

Two sequential samples from six aborting breeding cattle were collected, one sample was recovered within a month of abortion, and the other one six months after or prior to the abortion, whereas a unique serum sample from infected breeding cattle along gestation $(n=6)$ and noninfected non-aborting breeding cattle $(n=6)$ were analysed.

In addition, precolostral sera from calves born to $N$. caninum infected $(\mathrm{n}=4)$ and non-infected dams $(\mathrm{n}=4)$ were also analysed. Samples were deemed to be seropositive if they had IFAT titers equal to or greater than 1:200.

Sera from experimentally-infected animals were obtained from the Moredun Research Institute (Edinburgh, Scotland). Animals had been infected with NC-1 isolate as previously explained (Innes et al. 2001). Briefly, the experiment was comprised of four groups of breeding cattle 
and three groups of calves born from breeding cattle groups II, III, and IV. Animals in groups III (infected with $N$. caninum prior to mating and challenged with $N$. caninum at mid-gestation) and IV (infected with $N$. caninum prior to mating and left unchallenged throughout pregnancy), had been inoculated with $10^{7} \mathrm{~N}$. caninum tachyzoites in a previous pregnancy and were inoculated with $10^{8}$ live $N$. caninum tachyzoites, subcutaneously over the left prefemoral lymph node six weeks prior to mating. Cattle in groups II (challenged with $N$. caninum at midgestation) and III were each challenged at day 140 of gestation with $5 \times 10^{8}$ live $N$. caninum tachyzoites subcutaneously over the right pre-femoral lymph node. A control inoculum $\left(5 \times 10^{6}\right.$ Vero cells $)$ was used to challenge cows in group I (uninfected controls). A summary of the samples analysed in the present work is shown in the Table II. Breeding cattle sera were obtained at four different times after challenge and for calves, two sequential serum samples were tested, precolostral and at day 5 after birth. All serum samples in this study were aliquoted and stored at $-80^{\circ} \mathrm{C}$ prior to testing for specific antibodies by IFAT and WB-p17.

Parasites - In vitro maintenance of NC-1 isolate of $N$. caninum, purification of tachyzoites for IFAT and soluble antigenic extract were done according previously described procedures (Alvarez-Garcia et al. 2002).

Preparation and purification of $p 17$ antigenic fraction - Whole soluble extract was obtained from tachyzoites (Alvarez-Garcia et al. 2003) and subjected to SDS-PAGE. Proteins were localized in gels by their molecular weight. Then the gel containing the protein of interest was cut into pieces and eluted by electrophoresis for 6-7 $\mathrm{h}$ at 10
$\mathrm{mA} /$ tube according to the manufacturer's instructions (Electro-Eluter Mod. 422, Bio-Rad Laboratories S.A., Alcobendas, Spain). After elution, the degree of purification was assessed simultaneously by SDS-PAGE combined with silver staining and Western blot using a $N$. caninum-positive bovine serum with an IFAT titer of 1:3200. Finally, proteins were quantified by comparison with standard concentrations of bovine serum albumin (BSA, Roche Molecular, Biochemical, Mannheim, Germany) in silver-stained SDS-PAGE gels.

IFAT test - Sera were analysed by IFAT in double serial dilutions starting at 1:50 using the previously described method (Trees et al. 1993). An IFAT titer of 1:200 was employed as a cut-off point.

WB-p 17 - WB based on the purified $\mathrm{p}-17$ fraction was carried out according to previously described procedures (Alvarez-Garcia et al. 2003). Equal amounts of this protein fraction were exposed to each bovine sample, which were employed at 1:25 dilution. The $\mathrm{p} 17$ recognition by the different sera samples was quantified using densitometry (Fluor-S ${ }^{\mathrm{TM}}$ Multimager, Bio-Rad Laboratories) and expressed as OD values. Quantity One ${ }^{\circledR}$ quantitation software (Bio-Rad Laboratories) was employed for the image analysis.

Statistical analysis - Differences in IFAT titers and OD values among the different groups of naturally-and experimentally-infected cattle studied (inter-group variations) were analysed by non-parametric Mann-Whitney $U$-test and Kruskal-Wallis H-test employing the STATVIEW package v 4.0 (Abacus Concept Inc., Berkeley, CA, US).

TABLE I

Naturally-infected cattle: groups and samples analysed

\begin{tabular}{|c|c|c|c|c|}
\hline \multicolumn{3}{|c|}{ Breeding cattle } & \multicolumn{2}{|c|}{ Precolostral calves } \\
\hline \multicolumn{2}{|c|}{ Infected } & Non-infected & Infected & Non-infected \\
\hline $\begin{array}{l}\text { Aborting cattle } \\
a \quad b\end{array}$ & Non-aborting cattle & & & \\
\hline 6 & 6 & 6 & 4 & 4 \\
\hline
\end{tabular}

$a$ : within a month of abortion; $b$ : 6 months prior to or after abortion.

TABLE II

Experimentally-infected cattle: groups and samples analysed

\begin{tabular}{|c|c|c|c|c|c|c|c|c|}
\hline \multicolumn{6}{|c|}{ Breeding cattle } & \multicolumn{3}{|c|}{ Calves } \\
\hline Group & Week 0 & Week 1 & Week 4 & Week 20 & Total & Precolostral & Colostral & Total \\
\hline I & 5 & 5 & 5 & 5 & 20 & 4 & 4 & 8 \\
\hline II & 6 & 5 & 6 & 6 & 23 & 6 & 6 & 12 \\
\hline III & 6 & 6 & 6 & 6 & 24 & 6 & 6 & 12 \\
\hline IV & 3 & 3 & 3 & 3 & 12 & ND & ND & ND \\
\hline Total & 20 & 19 & 20 & 20 & 79 & 16 & 16 & 32 \\
\hline
\end{tabular}

Group I: uninfected controls; group II: challenged with Nespora caninum at mid-gestation; group III: infected with $N$. caninum prior to mating and challenged with $N$. caninum at mid-gestation; group IV: infected with $N$. caninum prior to mating and left unchallenged throughout pregnancy; weeks after challenge at day 140 of gestation; ND: not done 
To evaluate the effect of both variables - time and group - on antibody levels expressed as OD values, a parametric bifactorial Anova test was applied to the repetitive measures of antibody levels along time for all experimentally infected groups included in the study, and statistical calculations were computed using SAS v8.2. package (SAS Institute, Cary, NC, US). In this way, three null hypothesis $\left(\mathrm{H}_{\mathrm{o}}\right)$ were defined: $\mathrm{H}_{\mathrm{o}}{ }^{1}$ : The variable "group" has no effect on antibody levels; $\mathrm{H}_{\mathrm{o}}^{2}$ : There are no antibody fluctuations over time; $\mathrm{H}_{\mathrm{o}}^{3}$ : All groups of cattle have similar antibody fluctuations over time.

The degree of agreement between IFAT and WB-p17 was estimated by calculating the kappa value (Thrusfield 1995).

\section{RESULTS}

Antibody profiles in naturally-infected cattle - Specific antibodies were detected by both serological tests in all samples from aborting and non-aborting infected breeding cattle (Fig. 1), whereas no positive results were obtained in negative controls. Although higher OD values and higher IFAT titers were found in samples collected in the month of abortion than in samples collected six months before or after the abortion in breeding cattle, there was no statistically significant difference between both samplings.

When differences between aborting and non-aborting dams were investigated, the intensity of recognition detected in the WB-p17 was higher in naturally-infected breeding cattle in the month of abortion $(\mathrm{n}=6)$ compared with non-aborting breeding cattle $(\mathrm{n}=6)(P<0.05$, MannWhitney $U$-test). Using IFAT, these differences were not statistically significant $(P>0.05$, Mann-Whitney $U$-test). When samples taken 6 months before or after the abortion were included in the comparison $(n=12)$ the differences observed between infected aborting and infected non-aborting cattle were not significant $(P>0.05$, MannWhitney $U$-test). When pre-colostral samples were tested, both serological tests discriminated well between positive and negative samples since all congenitally infected calves proved to be seropositive by both tests (Fig. 1).

Antibody profiles in experimentally-infected cattle In breeding cattle OD values were present at high levels in groups III and IV during the entire sampling period. On the contrary, in group II recognition of p17 was not detected at weeks 0 and 1 following challenge, whereas moderate OD values were observed from week 4 after challenge at day 140 of gestation, reaching a high level at week 20 near parturition (Figs 2-3). This situation was

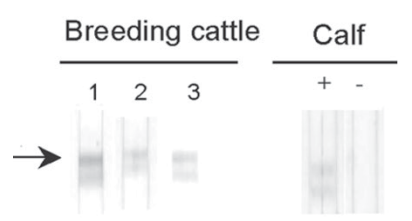

Fig. 1: recognition of the $\mathrm{p}-17$ protein fraction by specific $\operatorname{IgG}$ antibodies in cattle naturally-infected with Neospora caninum. 12: aborting cow. 1: month of abortion; 2: six months before or after abortion; 3: infected breeding cattle. The recognition of the p-17 protein fraction is indicated with an arrow. similar when IFAT results were analysed. The results obtained when the parametric bifactorial Anova test was employed for comparing antibody fluctuations detected in the three infected groups permitted to reject the three null hypotheses previously stated. The antibody responses clearly depended on the group considered $(P<$ 0.0001 ), rejecting $\mathrm{H}_{\mathrm{o}}{ }^{1}$. There were significant antibody fluctuations over time (rejecting $\mathrm{H}_{\mathrm{o}}{ }^{2}$ ), which varied between groups (rejecting $\mathrm{H}_{\mathrm{o}}{ }^{3}$ ). Antibody fluctuations were statistically significant between weeks 0 and 1 and weeks 4 and $20(P<0.0001$, Anova test). Differences in OD values and IFAT titers were statistically significant for each week postinfection among the three groups of infected breeding cattle $(P<0.0001$, Kruskal-Wallis $H$ test), except for week 20 in IFAT test as all groups showed similar high antibody titers.

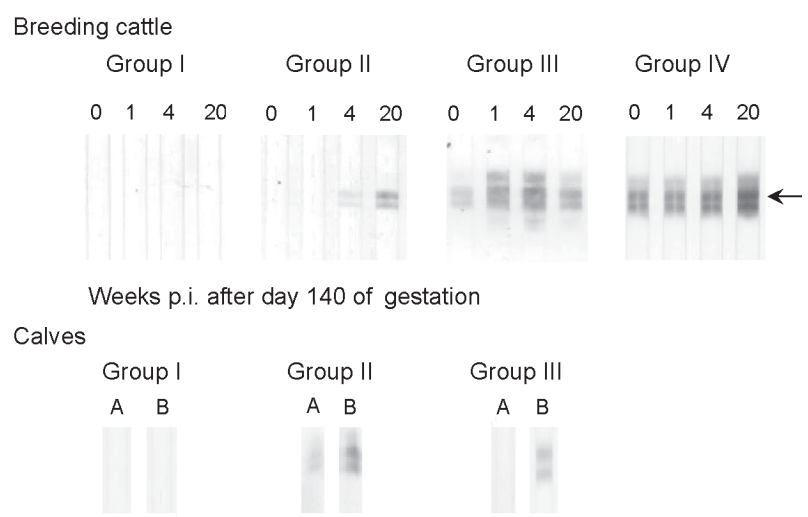

Fig. 2: recognition of the $\mathrm{p}-17$ protein fraction by specific $\operatorname{IgG}$ antibodies in four groups of cattle experimentally-infected with Neospora caninum. A: precolostral sample; B: colostral sample. The recognition of the $\mathrm{p}-17$ protein fraction is indicated with an arrow.

The WB-p17 discriminated well between positive and negative serum samples from calves born from experimentally-infected cows. Colostral antibodies were detected in groups II and III. Vertical transmission was only evident in calves from group II as antibodies against $N$. caninum were detected in precolostral sera by both tests (Fig. 3). Significant differences in antibody levels between two samplings in newborn calves were observed depending on the group and time of sampling $(P<0.0001$, Anova test).

Agreement between IFAT and WB-p17 - The antibody profiles determined by IFAT were compared with those obtained with the purified p17 for naturally-and experimentally-infected cattle. High agreement values were obtained when all bovine subpopulations were included in the comparative study $(\kappa=0.86)$, as well as for groups of breeding cattle $(\kappa=0.78)$ and calves $(\kappa=0.89)$ when considered separately.

\section{DISCUSSION}

The immunodominant nature of the 17 suggests that this antigen could be an ideal candidate for the develop- 
A
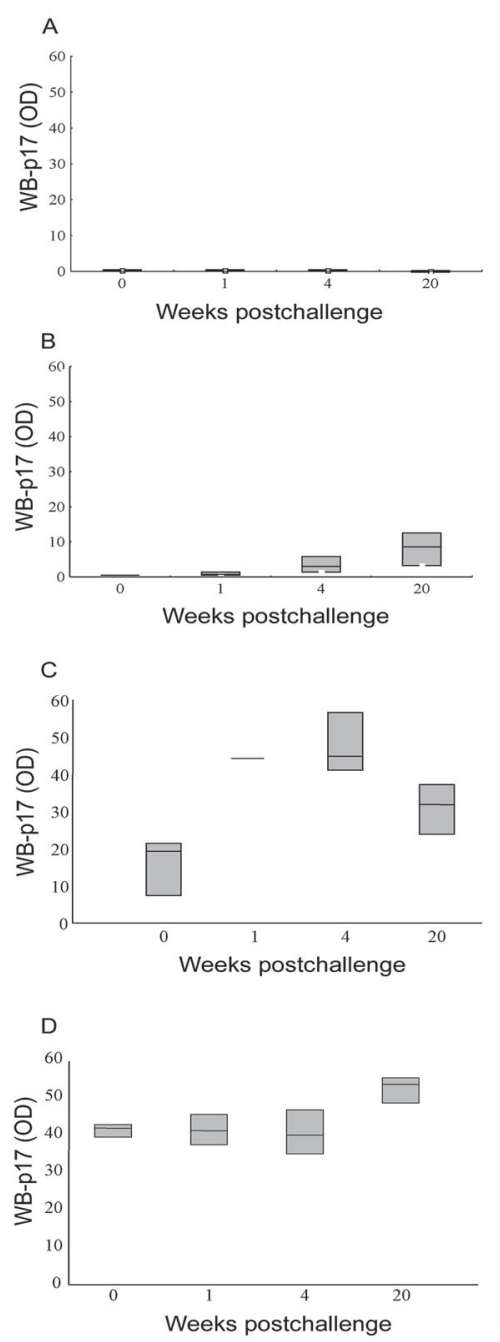

Weeks postchallenge
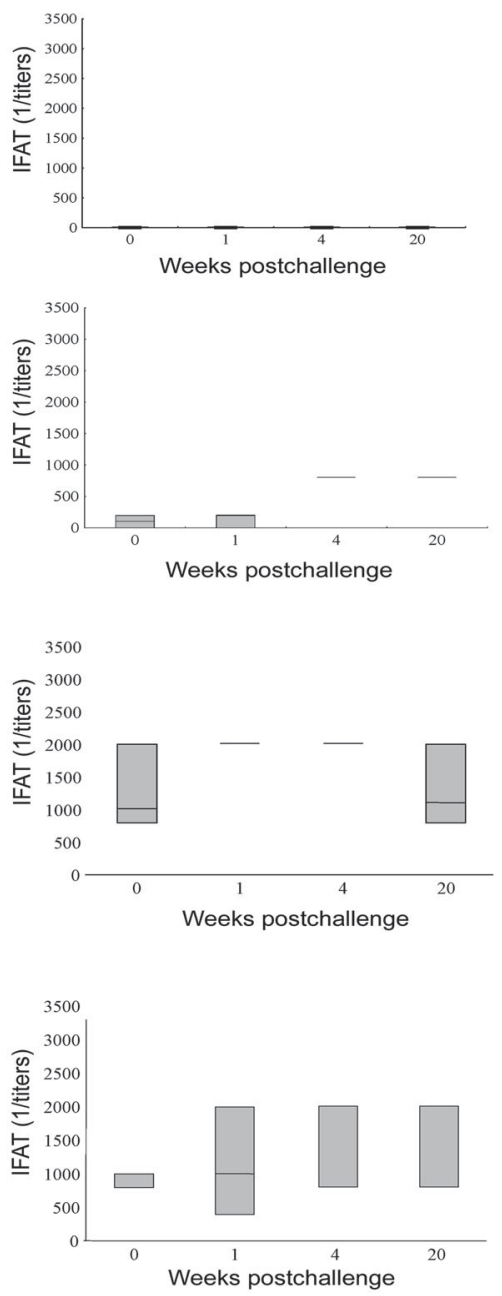

Weeks postchallenge

Fig. 3: box-plot graphs represent the lower, upper quartiles and median WB-p17 OD values and IFAT titers at different times after challenge at day 140 of gestation in four groups of cattle experimentally-infected with Neospora caninum. A: group I; B: group II; C: group III; D: group IV.

ment of serological diagnostic tests, since this antigenic fraction showed the highest frequency of recognition in naturally-infected breeding cattle and foetuses compared with other IDAs. The purified p17 was successfully employed in a quantitative diagnostic WB using densitometry to detect $N$. caninum- specific antibodies in sera. In our case, purified p17 was employed in a WB, following the procedure used in toxoplasmosis by Meek et al. (2003). Moreover, similar approaches have been previously reported for infectious diseases such as nocardiosis (VeraCabrera et al. 1992), where WB tests based on specific purified antigens were developed to investigate promising candidates for immunodiagnosis.

Several $N$. caninum-specific antigens have been employed in ELISA tests to diagnose $N$. caninum infection, proving to be highly sensitive and specific in the diagnosis of $N$. caninum infection in adult cattle. However, at present, the p-17 IDA has not been employed in the serodiagnosis of neosporosis.

The results obtained in the present study support the idea that this antigen could be a good candidate for the development of serological diagnostic tests as p-17 was detected in all infected cattle and differences in antibody levels among groups as well as fluctuating antibodies along time were detected by WB-p17 in a similar way as those use in other valuable tests. Furthermore, the requirements considered in the present study -the origin of the infection (naturally- or experimentally-infected cattle), the purpose of testing, sequential samples and different age groups- are desired when investigating the diagnos- 
tic value of an antigen (Jacobson 1998, Greiner \& Gardner 2000). As found in recent works (Gaturaga et al. 2005, Jenkins et al. 2005) sera from cattle infected by others biological related protozoa were not examined to test crossreactivity due to several reasons. Firstly, no crossreaction has been observed between Neospora infection and Toxoplasma, Cryptosporidium, Sarcocystis spp., Eimeria spp. or Babesia spp. infection in cattle (Schares et al. 2000, Howe et al. 2002) when ELISA tests based on crude extract or recombinant proteins were developed to detect Neospora specific antibodies in cattle. Moreover, a few cross reactions between the most closely related species $-N$. caninum and $T$. gondii - have been detected in WB corresponding to a few number of antigens whose apparent molecular weight is higher than $29 \mathrm{kDa}$ (Louie et al. 1997).

Initially, regarding the origin of the infection, p17 proved to have diagnostic value using sera from both natural and experimental infections. We found similar results using both techniques (IFAT and WB-p17) with samples from naturally-infected breeding cattle. In aborting breeding cattle higher IFAT titers as well as higher OD values were found in samples taken in the month of abortion, which is in accordance with the observations made by Quintanilla-Gozalo et al. (2000).

When differences between aborting and non-aborting naturally-infected breeding cattle were studied OD values were directly related with IFAT titers. Higher antibody levels were observed in aborting dams by both serological tests and our results agree with those obtained by others (Schares et al. 1998, Quintanilla-Gozalo et al. 2000). The statistically significant differences were only obtained with the WB-p17, so that according to our preliminary results future improvements of the assay based on $\mathrm{p} 17$ should involve optimisation of conditions, since one of the requirements of a serological test is to discriminate dams at risk of abortion from those infected dams without reproductive failure.

The results obtained for experimentally-infected breeding cattle are mainly in accordance with those reported in a previous work (Innes et al. 2001). The analysis of serial samples of breeding cattle throughout pregnancy permitted the detection of fluctuations in the IgG response directed against $\mathrm{p} 17$. In the animals in group II, which transmitted the infection to their descendents, antibody levels increased from week 4 after infection and remained lower than in the other breeding cattle groups mentioned above. The results from group III showed an increase of antibodies after the first week of reinfection, which remained high until parturition and in group IV, which did not transmit infection to the offspring, levels of antibodies remained high throughout the period of study until parturition. On the other hand, the differences observed in week 0 between groups III and IV may probably be due to individual differences since both groups are expected to present similar immune response. In general, fluctuations of antibodies detected by both tests in all groups are similar to those reported by others (Innes et al. 2001). The high recognition of $\mathrm{p} 17$ detected by sera from naturallyand experimentally-infected breeding cattle may be due to the fact that antibodies developed against p17 are a major specific IgG antibody fraction.

The $\mathrm{p} 17$ also permitted the detection of antibodies in precolostral sera which may be an important consideration in control programs of vertical transmission (Thurmond \& Hietala 1995). Our results confirm the diagnostic value of $\mathrm{p} 17$, previously pointed out by other authors (Louie et al. 1997).

The potential value of $\mathrm{p} 17$ in serological diagnosis is also supported by the high agreement values obtained between IFAT and WB-p17 for the different groups included in the comparative study. Variable agreement results have been obtained even when comparing similar serological tests (von Blumroder et al. 2004).

The use of p17 in serological tests may offer advantages over whole tachyzoite soluble extract, since antibodies directed against this protein represent a major specific IgG fraction in infected animals. The reactivity of bovine infected sera against the reduced antigen was easily detectable, indicating that this highly immunogenic antigen could be an optimal marker to be employed in an ELISA test based on a recombinant antigen.

In future studies, an ELISA test utilizing this antigen alone or in combination with other specific antigens may help us to improve the sensitivity, specificity and scope over existing diagnostic tests.

\section{ACKNOWLEDGEMENTS}

To Centro de Cálculo (UCM), specially to Ricardo García de la Mata, for the statistical analysis. To Vanessa Navarro and Raquel Expósito for their excellent technical assistance.

\section{REFERENCES}

Alvarez-Garcia G, Collantes-Fernandez E, Costas E, Rebordosa X, Ortega-Mora LM 2003. Influence of age and purpose for testing on the cut-off selection of serological methods in bovine neosporosis. Vet Res 34: 341-352.

Alvarez-Garcia G, Pereira-Bueno J, Gomez-Bautista M, OrtegaMora LM 2002. Pattern of recognition of Neospora caninum tachyzoite antigens by naturally infected pregnant cattle and aborted foetuses. Vet Parasitol 107: 15-27.

Bjerkas I, Jenkins MC, Dubey JP 1994. Identification and characterization of Neospora caninum tachyzoite antigens useful for diagnosis of neosporosis. Clin Diagn Lab Immunol 1: 214-221.

Dubey JP 1999. Neosporosis - The first decade of research. Int J Parasitol 29: 1485-1488.

Gaturaga I, Chahan B, Xuan X, Huang X, Liao M, Fukumoto S, Hirata H, Nishikawa Y, Takashima Y, Suzuki H, Fujisaki K, Sugimoto C 2005. Detection of antibodies to Neospora caninum in cattle by enzyme-linked immunosorbent assay with truncated NcSRS2 expressed in Escherichia coli. $J$ Parasitol 91: 191-192.

Greiner M, Gardner IA 2000. Epidemiologic issues in the validation of veterinary diagnostic tests. Prev Vet Med 45: 322.

Howe DK, Tang K, Conrad PA, Sverlow K, Dubey JP, Sibley LD 2002. Sensitive and specific identification of Neospora caninum infection of cattle based on detection of serum antibodies to recombinant Ncp29. Clin Diagn Lab Immunol 9: 611-615. 
Innes EA, Wright SE, Maley S, Rae A, Schock A, Kirvar E, Bartley P, Hamilton C, Carey IM, Buxton D 2001. Protection against vertical transmission in bovine neosporosis. Int J Parasitol 31: 1523-1534.

Jacobson RH 1998. Validation of serological assays for diagnosis of infectious diseases. Rev Sci Tech 17: 469-526.

Jenkins MC, Fetterer R, Schares G, Bjorkman C, Wapenaar W, McAllister M, Dubey JP 2005. HPLC purification of recombinant NcGRA6 antigen improves enzyme-linked immunosorbent assay for serodiagnosis of bovine neosporosis. Vet Parasitol 131: 227-234.

Louie K, Sverlow KW, Barr BC, Anderson ML, Conrad PA 1997. Cloning and characterization of two recombinant Neospora protein fragments and their use in serodiagnosis of bovine neosporosis. Clin Diagn Lab Immunol 4: 692699.

Meek B, Diepersloot RJ, van Gool T, Speijer D, Peek R 2003. IgM recognition of recombinant Toxoplasma gondii antigens by sera of acutely or latently infected humans. Diagn Microbiol Infect Dis 45: 45-52.

Quintanilla-Gozalo A, Pereira-Bueno J, Seijas-Carballedo A, Costas E, Ortega-Mora LM 2000. Observational studies in Neospora caninum infected dairy cattle: relationship infection-abortion and gestational antibody fluctuations, In A Hemphill, B Gottstein (eds), A European Perspective on Neospora caninum. Int J Parasitol 30: 900-906.
Schares G, Peters M, Wurm R, Barwald A, Conraths FJ 1998. The efficiency of vertical transmission of Neospora caninum in dairy cattle analysed by serological techniques. Vet Parasitol 80: 87-98.

Schares G, Rauser M, Sondgen P, Rehberg P, Barwald A, Dubey JP, Edelhofer R, Conraths FJ 2000. Use of purified tachyzoite surface antigen p38 in an ELISA to diagnose bovine neosporosis. Int J Parasitol 30: 1123-1130.

Thrusfield M 1995. Diagnostic testing. Veterinary Epidemiology, The University Press, Cambridge, p. 266-285.

Thurmond M, Hietala S 1995. Strategies to control Neospora infection in cattle. The Bovine Practitioner 4: 29-32.

Trees AJ, Guy F, Tennant BJ, Balfour AH, Dubey JP 1993. Prevalence of antibodies to Neospora caninum in a population of urban dogs in England. Vet Rec 132: 125-126.

Vera-Cabrera L, Salinas-Carmona MC, Welsh O, Rodriguez MA 1992. Isolation and purification of two immunodominant antigens from Nocardia brasiliensis. J Clin Microbiol 30: $1183-1188$

von Blumroder D, Schares G, Norton R, Williams DJ, EstebanRedondo I, Wright S, Bjorkman C, Frossling J, Risco-Castillo V, Fernandez-Garcia A, Ortega-Mora LM, Sager H, Hemphill A, van Maanen C, Wouda W, Conraths FJ 2004. Comparison and standardisation of serological methods for the diagnosis of Neospora caninum infection in bovines. Vet Parasitol 120: 11-22. 